\title{
IMPACT OF ARTIFICIAL INTELLIGENCE ON PROFESSIONAL AUTONOMY OF PATHOLOGISTS
}

By

\author{
Saman Feroze
}

Master of Science in Biochemistry, Aligarh Muslim University, 2008

Bachelor of Science in Biochemistry, Aligarh Muslim University, 2005

A thesis presented to Ryerson University

in partial fulfillment of the

requirements for the degree of

Master of Science in Management

in the program of

Master of Science in Management

Toronto, Ontario, Canada, 2020

(C) Saman Feroze, 2020. 


\section{AUTHOR'S DECLARATION}

I hereby declare that I am the sole author of this thesis. This is a true copy of the thesis, including any required final revisions, as accepted by my examiners.

I authorize Ryerson University to lend this thesis to other institutions or individuals for the purpose of scholarly research.

I further authorize Ryerson University to reproduce this thesis by photocopying or by other means, in total or in part, at the request of other institutions or individuals for the purpose of scholarly research.

I understand that my thesis may be made electronically available to the public. 


\section{Abstract \\ Impact of Artificial Intelligence on Professional Autonomy of Pathologists}

Saman Feroze, 2020

Master of Science in Management

Ryerson University

Advancements in Artificial Intelligence (AI) are paving the way to applications in pathology, with the hope of making significant contributions towards patient care. The prospect of implementation in clinical practice also raises organizational and ethical questions. Professional autonomy of pathologists is the freedom to make prognostic and diagnostic decisions independently that best meets the needs of patients. Researchers have highlighted some of the potential impacts of AI on professional autonomy. However, it is not clear how individual pathologists perceive its potential impact on their own practice.

The purpose of this study is to investigate the perspective of pathologists on the impact of Artificial Intelligence on their professional autonomy. The results of this study highlight the ethical concerns of pathologists related to decision making bias and their retroactive monitoring. Results also highlight the importance of curriculum and policy interventions to build upon technical knowledge and skills of pathologists and to have control over the process of validation and regulatory processes of AI tools and that these tools might make pathologists more objective and less mindful of resources. 


\section{Acknowledgements}

I hope this research takes a step towards bridging the gap between Artificial Intelligence and Healthcare. I owe thanks to several people who have made this endeavor possible.

First and foremost, I am sincerely grateful to my thesis supervisor Dr. Julien Meyer for his patience, immense knowledge contribution and continuous support throughout my master's program. I would also like to recognize his valuable assistance and his in-person involvement in the data collection process of interviewing pathologists besides the guidance and encouragement he provided in every step of this thesis.

I wish to express my sincere appreciation to my committee members who are subject matter experts, Dr. Chris MacDonald and Dr. April Khademi for taking out their valuable time to provide feedback on my thesis content and assist in its improvement.

I would like to pay my special regards to the respectful and kind pathologists who were more than willing to participate in the data collection process of this paper. This research thesis would not have been possible without their inclination towards sharing valuable knowledge and insights on the topic.

I wish to thank all the brilliant professors of Ryerson university and my fellow batchmates for their continuous backing and encouragement. I especially want to thank Rijuta Menon and Guneet Kaur for bringing beautiful colors of laughter and giggles even during the hardest of hard times and helping me navigate the realm of research and academia once more.

I wish to acknowledge the support and great love of my family, my husband, my mom and my dad, who left us for his heavenly abode in the middle of this journey. Finally, I would like to give a special shout out to my three beautiful daughters who have sacrificed the most with no complains. They kept me moving and this work would not have been possible without their support. Thank you all very much!! 


\section{Dedication}

This thesis is dedicated to my husband Khalid Liaqat. Without you I would never have had the courage to begin this journey. I am forever grateful for all your support and encouragement. Your confidence in me is my greatest strength. I Love you always. 


\section{TABLE OF CONTENTS}

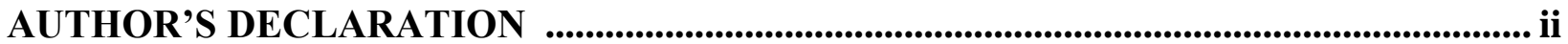

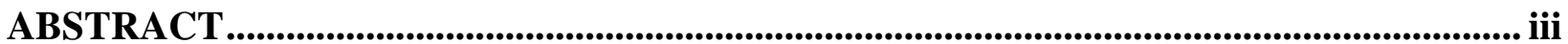

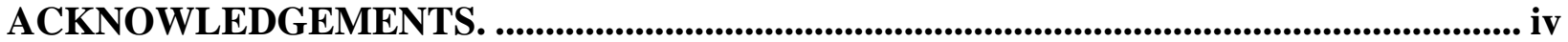

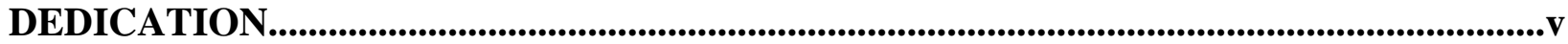

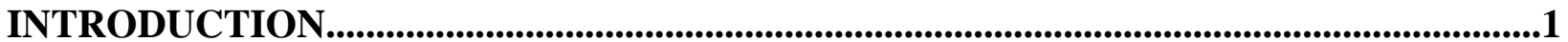

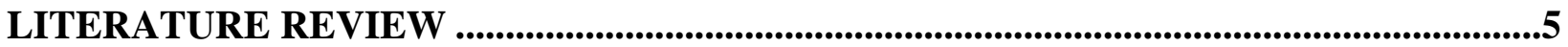

Professional Autonomy and its four dimensions ............................................................................. 5

Perceived Threat to Professional Autonomy .......................................................................7

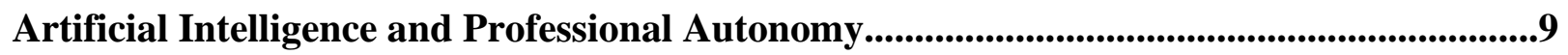

Advanced Knowledge and skills of pathologists and Artificial Intelligence ........................11

Ethical code of conduct and Artificial Intelligence ..........................................................13

Patient centered mindset and Artificial Intelligence ...........................................................15

Self-regulation and Validation of Artificial Intelligence tools...................................................16

METHODOLOGY ..................................................................................................................18

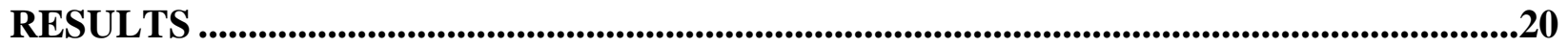

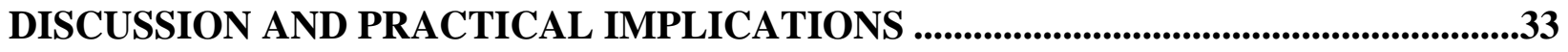

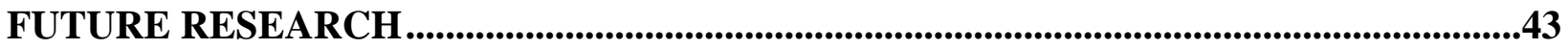

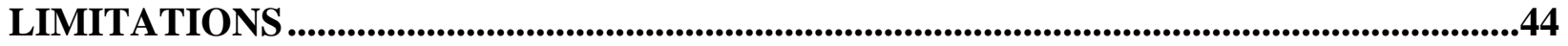

CONCLUSIONS

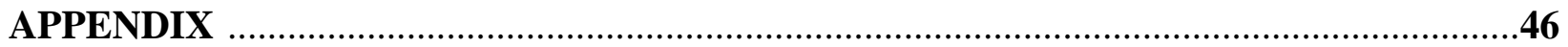

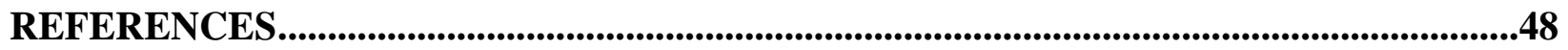




\section{Introduction}

Professional Autonomy is defined as the freedom to have complete individual and independent control and judgement over every aspect of the professional work including processes, procedures, content and application of one's professional body of knowledge and aptitude (Skar, 2010).

The term professional autonomy is used in World Medical Association of professionals where physicians around the globe meet. Officially entitled the WMA Declaration of Madrid on Professional Autonomy and Self-Regulation in 1987, the old Declaration of Madrid consisted of 10 Items regarding professional autonomy and self-regulation (Tezuka, 2014). The main points regarding professional autonomy described in the declaration reflect its core dimensions that need to be preserved as basic principles in medical ethics. These dimensions of professional autonomy are 1. Ethical Code of Conduct, 2. Advanced knowledge and skills, 3. Self-regulation, 4. Patient centered mind set. These are the fundamental features of a profession. All 4 features relate to the theme of this paper, professional autonomy (Tezuka, 2014) These four dimensions of professional autonomy are the key to differentiate professionals from non-professionals (Salvatore et.al, 2018).

Ethical conduct is a combination of values and standards for professionals (Baron, 1992). Ethical code of conduct are guidelines that are useful as a recommendation of proper conduct by medical professionals including pathologists. These guidelines are beneficial when pathologists are faced with ethical decisions in their practice. Pathologists practice by forming an organization or group and form code of ethics for their peers depending upon the standard of their profession in society (Baron, 1992). These codes may exert a powerful influence in defining and facilitating ethical practice by clearly stating expectations and responsibilities for pathologist's ethical conduct (Kenny et. al, 2010). This drives the pathologists to be more responsible towards their autonomous freedom to govern themselves and control their affairs by being the independent decision maker. 
As a result of this autonomy, pathologists enjoy clinical, social and economic and organizational work freedom (Salvatore, 2018).

Advanced knowledge and skills are a characteristic that is required by professionals in order to master a certain skill. This often requires them to carry out extensive training and long duration of education (Black-Schaffer et.al, 2016). This dimension of autonomy differentiates professionals from non-professionals and thus refers to a major reason why only professionals are given the right to autonomously practice their profession (Salvatore, 2018).

Regulation ensures that professional's practice in safe, competent and ethical manner. Selfregulation is a characteristic of professionals including pathologists to regulate their own conduct in order to prevent the patients from harm that may occur. Government grants permission to a group of professionals including pathologists to self-regulate their performance because of the specialized set of knowledge they possess (Tezuka, 2014). Self-regulatory body is a private professional body assigned by government based on their specialized body of knowledge and differs from government regulation. Government regulatory bodies provide license to professionals through public qualification exams which prohibits non-professionals from performing the acts of professionals (Collier, 2012).

According to national medical associations, professional autonomy is essential to the practice of pathology to provide high-quality results and thus it must be the priority for pathologists to enhance the benefit of patients (Tezuka, 2014). One of the basic principles in medical ethics is for medical professionals to assure professional autonomy at times of patient care. Based on this, professional autonomy makes pathologists keep a check on their professional conduct. Medical professionals including pathologists are given training to be more mindful, informative, and empathic which transforms their role from one characterized by authority to one that has the goals of partnership, 
solidarity, empathy, and collaboration (Epstein and Street, 2011). Thus, patient centered care is a dimension of professional autonomy and is important to enhance the quality of patient care. Pathologists build on their patient centered mindset because their primary objective is to contribute to the society by fulfilling the demands of patient care and not undertake their duties like a business transaction to produce profits (Tezuka, 2014). Therefore, patient centered mindset is crucial for pathologists to practice professional autonomy and improve the quality of patient care.

Physicians have reported greater professional autonomy and ability to provide more time to patients to be directly reflective of their job satisfaction level (Tyssen et. al, 2013).

This has been proved to be significant for different attributes of professional autonomy like freedom to make clinical decisions, high quality of care and greater job satisfaction of pathologists and our focus in this study would be on professional autonomy (Tyssen et. al, 2013, Walter and Lopez, 2008). AI tools operate by coding knowledge to improve decision making by pathologists and contribute as a threat to their professional autonomy. Clinical decision support systems are being used to improve the quality of decision making. CDSS is an AI based tool that is making use of large amounts of data and producing decision making insights for medical professionals (Walczak and Steven, 2018). According to Tizhoosh, the ultimate decision-making lies in the hands of medical professionals and when pathologists are solving many cases independently, it exposes them to variety of medical cases and strengthens their mode of knowledge gain (Tizhoosh and Pantanowitz,2018). Whereas when the decision making is done by new technological systems using knowledge codification, there is a great chance of knowledge being distributed to other subordinate professionals making pathologists lose the sole possession of unique medical knowledge and ultimately perceive technology as a threat to professional autonomy (Walter and Lopez, 2008). 
One such latest cluster of technologies focused on the ability of computers to make flexible rational decisions is Artificial intelligence. Artificial intelligence (AI) is defined as the science of making intelligent machines that aim to imitate human cognitive functions (Hamet and Tremblay, 2017). In healthcare, as the availability of data is vast and rapidly increasing, it has become more feasible to train the AI systems by using sophisticated algorithms and then deploy it in healthcare applications (Tizhoosh and Pantanowitz, 2018). Altogether, AI techniques have initiated a very active discussion of whether medical pathologists and their power of professional autonomy will be ultimately replaced by AI systems in the future (Serag et.al, 2019).

There has been a significant rise in the number of researches conducted on the use of algorithms and machine learning tools by pathologists to detect different kinds of cancers. Currently, AI tools are not used in routine, clinical practice by pathologists, but are actively used for research purposes only (Serag et.al, 2019). Therefore, the focus of this study is to explore the perception of pathologists in integrating and using these tools daily as a part of their daily pathology practice. 


\section{Literature Review}

The literature review focuses on the four dimensions of professional autonomy in detail, followed by perceived threat to professional autonomy. It also includes the role of Artificial Intelligence in pathology and in relation to the different dimensions of professional autonomy.

\section{Professional autonomy and its four dimensions}

Professional autonomy is the freedom to make diagnostic and prognostic decisions objectively with complete responsibility and accountability for reports sent out. The concept of Professional autonomy could be approached in two ways. The first one is called sociological autonomy, which determines the internal power linked to the image of professionals especially physicians in the health care system (Esmaeilzadeha et. al, 2015). This approach highlights the importance of professional power in the society and its consequences. The other approach called as normative professional autonomy "considers professional autonomy as an ideal that is related to a standard of excellence for each profession including medical professions comprising of personal services that are marked by confidentiality. This approach implies that professional autonomy of physicians can be maintained only if they allow their activities and decisions to be peer reviewed by other physicians and by patients" (Esmaeilzadeha et. al, 2015).

The importance professional autonomy holds for pathologists makes them practice their profession with a lot of responsibility and prevents them from being cavalier in their clinical judgement (Young, 2017). Pathologists sign the report only when they have given their best clinical diagnosis without getting influenced by any physicians, resident or technology. However, they do not work in isolation and if the information received by pathologist to give diagnosis is not complete, they get additional information from the respective departments and in case of doubt they also take 
second opinions from colleagues before signing the report as the responsibility is solely on their shoulders (Jeffery et al., 2008)

"In medicine, professional autonomy refers to the freedom of medical professionals to exercise professional judgment in the care and treatment of their patients, to be regulated by self-governing, professional medical bodies, and to advocate on behalf of patients and society"(Doctors of BC,p.1, 2016). Professional autonomy does not mean absolute freedom, but rather, it lies on a continuum and comes with responsibilities. In order to be granted considerable autonomy and the privilege of self-regulation, medical professionals including pathologists are devoted to serving the best interests of society by providing high quality evidence informed care (Doctors of BC, p.1,2016). Similarly, Immanuel Kant in 1788 postulated the term autonomy. According to Kant, "Physicians impart medical care to their patients with spirit of positive freedom or in accordance to ones established standards without external governance" (Hashimoto, p. 2, 2006). Therefore, professional autonomy is an autonomous right that is enjoyed by the professionals by voluntarily forming their own code of ethics and enthusiastically following them as well. This describes the legitimacy of this positive freedom of autonomy specifically for professional careers including physicians and pathologists (Hashimoto, 2006). Pathologists are given the right to make autonomous decisions based on the four dimensions of professional autonomy. According to Kazuo (2014), the four fundamental dimensions of professional autonomy are 1. Ethical Code of conduct 2. Advanced knowledge and skills, 3. Self-regulation, 4. Patient centered mind set. These four dimensions form the thematic framework of this paper and are discussed in the next sections of literature review. 


\section{Perceived Threat to Professional Autonomy}

"Perceived threat to Professional autonomy is the degree to which a person believes that using a particular system would increase or decrease his or her control over the conditions, processes, procedures, or content of his or her work" (Walter and Lopez, 2008). Threat to professional autonomy is a salient outcome belief affecting the pathologist's acceptance of new IT systems (Walter and Lopez, 2008) Usefulness of technological tools and intention to use these tools are both negatively correlated to the perceived threat to professional autonomy of pathologists (Walter and Lopez, 2008).

Threat to professional autonomy highlights several aspects of fear and anxiety faced by pathologists of losing the value of different dimensions of professional autonomy, their capability of self-regulating their performance, the ethical code followed by the pathologists and the coworkers from their organization and the patient centered mind set of pathologists. Some researchers have pointed out that pathologists are reluctant to deploy Artificial intelligence tools in digital pathology and "resist revalidation policies that require them to collect data, claiming it's too demanding or expensive or that it detracts from patient care" (Collier, p. 2, 2012). Pathologists are more concerned about trust of patients and physicians on their skill set because their mind set have long valued the patient well-being as the very center of the health care system (Young, 2017, Tizhoosh and Pantanowitz, 2018, Hamet and Tremblay, 2017). Any IT system that operates by coding knowledge to improve decision making originally practiced by pathologists can contribute as a perceived threat to their professional autonomy (Walter and Lopez, 2008). Electronic medical record EMR is an AI based system used extensively in healthcare. Use of AI based systems has proved to be beneficial in enhancing patient care and work performance of medical professionals in specializations including pathology (Ahuja, 2019). One of the studies showed that $65 \%$ of 
physicians indicated that use of EMR has improved patient care which is suggestive of the positive impact it has brought in the work life of medical professionals (Manca, 2015). The introduction of machines in the $19^{\text {th }}$ century left many workers across different occupations redundant; this has also inculcated fear in the minds of health care professionals that AI will become a substitute to human decisions or worse replace them completely in the future (Scott, 2018). One of the reasons for these fears is due to the powerful AI techniques that can assist in decision making by cracking the relevant information hidden in the vast amount of healthcare data (Jiang et.al, 2017).

For physicians, including pathologists, to successfully adapt and implement artificial intelligence it is very important for them and other users involved in the healthcare system to accept it and show their complete trust and involvement in its implementation.

It has been made clear in past studies (Hosny et. al, 2018) that AI provides clinical insights that does not stem from traditional sources of professional authority and is thus perceived as a threat to physicians' decision autonomy. But was this threat a concern for all the dimensions of professional autonomy as well? The answer is not clear. Therefore, the goal of my research is to explore the perception of pathologists on how, according to them, artificial intelligence is going to impact the four dimensions of professional autonomy in order to keep a check on what is taken by pathologists as a challenge or enthusiasm in adopting these tools.

There are certain elements of technology that are considered by pathologists as threats to their professional autonomy. Physicians are often motivated to use IT systems for their clinical activities by organizations they work for as a means of improving patient care (Salvatore et. al, 2018) Threat to professional autonomy is found to be negatively correlated with the intention of physicians to use decision support systems in public and private hospitals. Knowledge codification function of Clinical decision support system (CDSS) and EMR are seen to negatively impact the intention of 
pathologists on using these tools as it was considered a threat to their professional autonomy. (Esmaeilzadeh et.al, 2015, Walter and Lopez, 2008). Similarly, other studies documented resistive attitude of healthcare professionals in accepting new technology like telemedicine and EMR, if there was any negative consequence or harm coming to their autonomy (Xue et.al, 2015, Albert and Manda et.al, 2010).

In the upcoming sections I will be discussing Artificial intelligence and professional autonomy in terms of its four dimensions--advanced knowledge and skills, ethical code of conduct, patient centered mindset and self-regulation by pathologists.

\section{Artificial intelligence and Professional autonomy}

"Artificial intelligence refers to intelligence represented by some artificial entities like robots and computers that are aimed at approximating some aspect of human cognitive thinking" (Chang et.al, p.1, 2019). AI has received a lot of recognition in the past few years specially in digital pathology because of its unique capability of visual object and image recognition (Chang et. al, 2019). The pathological benefits of AI are expanding in all sectors of healthcare from tissue image analysis for diagnosing cancer to morphological analysis and prognosis prediction. Papanicolaou test imaging, approved by the US Food and Drug Administration (FDA) for screening purposes, is now fully used (Timothy, 2017). Huge amount of research studies has shown the success of AI tools in increasing the diagnostic treatment accuracy and efficiency (Tizhoosh and Pantanowitz, 2018). It is now evident that AI tools will be a tool for pathologists and not a replacement (Timothy, 2017). Some of the benefits of AI tools in pathology are related to saving time of pathologists by doing the repetitive tasks of screening, speeding up the process of disease diagnosis and reducing the errors in pathology reports by highlighting the mistakes are a few to mention (Tizhoosh and Pantanowitz, 2018). However, besides the technical diagnostic benefits of using AI tools in 
pathology, these tools not getting very actively implemented in the daily practice of pathologists. The success of pathologists will depend on the successful implementation of these tools (Timothy, 2017). This paper is focused on exploring the impact of AI on independent decision-making power referred to as professional autonomy of pathologists.

Professional autonomy as mentioned earlier is a very important aspect of every health professional's work life because it imprints independent decision-making power in them and gives them the confidence to exercise individual judgements. One can refer it as the "heart of their profession". Health professionals have been adopting and implementing new IT systems in the past and have faced their share of benefits and challenges. Medical professionals including radiologists and pathologists are readily embracing AI to use its potential benefits (Hosny et.al, 2018). However, there is always a varied user perception and a balance must be set between AI and human intelligence to enjoy the combined benefits of both that neither of them can do alone (Holzinger et.al, 2018). Research reveals that technology adoption behavior of physicians depends on how technology is impacting their professional autonomy (Lin, C., Lin, I., \& Roan, J. ,2012). The focus of this study was on the barriers, perceived threat and inequity issues in adopting latest technology. 115 physicians participated in a questionnaire survey and the results show that if technology is taken to be a perceived threat by physicians it has a negative impact on their behavioral intention to use that IT system and is perceived by them as less useful. The research finding presented a better insight into physicians' rejection and the antecedents of such outcome. For the healthcare industry understanding the factors contributing to physicians' technology acceptance is important as to ensure a smooth implementation of any new technology. The results of this study also provided change managers reference to a smooth IT introduction into an organization (Lin, C., Lin, I., \& Roan, J. ,2012). 
Tredinnick argues that many professionals including pathologists take AI as a threat due to their belief that the new AI system will interfere in their natural stream of work routine and practice patterns (Tredinnick ,2017). Also, in another article review the author is of the opinion that pathologists are worried about their knowledge like diagnosis made on specific images being organized, codified and leaked to peers and non-professionals (Tizhoosh and Pantanowitz, 2018). Physicians being professionals do not feel comfortable being directed, advised and instructed by an Artificial IT system (Sambasivan, 2012) All these factors together may have embedded in the brains of some medical professionals that AI can take over them, their professional identity and knowledge in the future (Sambasivan, 2012).

\section{Advanced knowledge and skills of pathologists and Artificial Intelligence}

Decision making by healthcare providers including pathologists involves many kinds of expert knowledge and skills like scientific knowledge concerned with explanation of symptoms and disease, technological knowledge and skills that assist in disease mitigating and cure and implicit knowledge of diagnosis and care (Nordin, 2000). Daily activities of a pathologist include visualizing the slides of human tissue under a microscope and grading the tissue depending upon the severity of the diagnosis, followed by final diagnostic report that is released and then used by physicians for diagnosis, treatment decisions and prognosis (Kayser, 2012). One very important aspect of physician's knowledge and skills is their continuous practice and frequency of clinical exposure. If a physician is not actively practicing his profession, decay of knowledge and skill sets in (Yang et. al, 2012). Technology implementation is known to extend a helping hand across professions and therefore professionals including pathologists are looking to utilize technological tools like Artificial Intelligence in their daily practice. Artificial intelligence works by applying

pattern recognition to relevant signs and symptoms in digital images of tissues of patients to 
improve diagnostic accuracy and efficiency. AI technology innovations are bringing a paradigmatic shift in health care, where physicians need to learn how to process the data meaningfully and efficiently. For the AI tools to be implemented in pathology, computer uses an algorithm to build experience from large amounts of training data of digital pathology images (Salla et. al, 2018). In this scenario, what happens is that over a period, pathologists will be prevented from cumbersome workload of making glass slides and visualizing them with light microscopy but at the same time this will also lead to the deterioration of the clinical and cognitive skills of pathologists due to lack of regular practice (Salla et. al, 2018). It can also be claimed that AI will reduce the knowledge gap between professionals and nonprofessionals by deteriorating the advanced knowledge and skills of physicians and also by sharing the knowledge whose sole owners are physicians among other co-workers. A literature review was recently published in which the author explains the impact of artificial intelligence on medical expertise and concluded that AI can negatively affect doctor-patient communications, physical examination skills, and development of clinical knowledge which are critical for making diagnosis (Salla et. al, 2018). This loss in advanced theoretical knowledge and technical skills can be perceived as a threat to professional autonomy as it reduces the skills and knowledge of physicians. Also, IT systems including AI functions by codification of expert knowledge and store it in the form of data that can be saved and spread to other coworkers or paraprofessionals thus making the medical professionals lose the possession of this unique advanced set of knowledge and skills. Use of AI tools could also provide insight to non-professionals about the unique practice of medical profession thus weakening the professional autonomy of physicians (Walter and Lopez, 2008). As a result, physicians claim of possessing expertise over other workers is invalidated which in turn weakens their professional autonomy. 


\section{Ethical code of conduct practiced by pathologists and Artificial Intelligence}

Medical ethics focuses primarily on issues arising from medical practice of medical professionals including pathologists (Holm, 2006). The ethical assessment in healthcare is based on four principles of medical ethics introduced by Beauchamp and Childress- autonomy, non-maleficence, beneficence, and justice (Page, 2012). These have been extremely influential in the field of medical ethics and are fundamental to understanding the current approach to ethical assessment in health care. Respect for autonomy refers to acting intentionally after being given sufficient information and time to understand the information. Beneficence is directed to promote the well-being of patients and society. On the other hand, non-maleficence implies first do no harm which can be achieved by careful decision making and having adequate training. Justice is another component of ethical dimension and deals with the equitable distribution of social benefits (Avasthi et.al, 2013). "Global ethical code for all physicians" approved by the World Medical Association (WMA) at its general annual assembly held in Chicago in 2017 states that physicians are to respect the "autonomy and dignity" of patients. The pledge also calls for doctors to keep patient information confidential, even after their deaths, and to share medical knowledge to advance health care and benefit patients (CMAJ, 2017). Practicing the medical ethics and making sure that the coworkers practice as well in their routine professional life is a very important aspect of physicians profession (Tezuka, 2018 and Hashimoto, 2006), breach of these regulations is considered a valid threat to their professional autonomy and is one of the major reasons behind ethical complications on the physician-patient relationship that have emerged particularly on the issues of autonomy (Norman et. al, 2011).

Data Science has become an integral part of health care delivery system. The technological advances in health care require collecting and sharing of massive amount of data (Noorbaksh, 
2019). Data science including AI pose various ethical challenges like the ethics of data and privacy, ethics of algorithms and morality and ethics and values of practice. Among these, privacy has been the center of attention. Privacy is defined as a fundamental human right in the Universal Declaration of Human Rights at the 1948 United Nations General Assembly (Noorbaksh, 2019). Artificial intelligence plays a very important role in digital pathology by pattern or image recognition and thus require large amount of labelled data sharing among the interested organizations needing access to the images either for the purpose of training or decision making. This leads to an increase in the number of companies which gain access to the health data and can use it illegally or unethically (Tizhoosh and Pantanowitz, 2018). Several studies have proved the use of technology such as electronic medical record (Norman et.al, 2011 and Ozair et al, 2015) and artificial intelligence applied in health care to violate the ethical code of conduct of physicians by challenging the data governance mechanisms and systems comprising of patient medical records, diagnosis or annotated tissue images (Keskinbora, 2019). This reduces the trust of patients in pathologists and weakens the patient-physician bond as patients are not sure if their privacy is intact with them.

\section{Patient centered mind set of pathologists and Artificial Intelligence}

The term "altruism" was introduced as an opposite to "egoism" by the nineteenth century French philosopher Auguste Comte as a guide to working in the interests of others. With its sense of service and self-sacrifice, altruism is often thought to be an integral factor of medical professionalism. Medical professionals including pathologists are granted the monopoly of practicing their profession with autonomy on the understanding that they will guarantee their services with altruism, morality and integrity to provide their best services for patient care and well-being (Cruess et. al, 2004). For pathologists this means consistently placing the interests of 
individual patients and society above their own and putting profits second (Cruess et.al, 2004). Harris states unequivocally that patient centered altruistic mindset is a necessary part of the medical profession. "Values such as compassion, altruism, integrity, and trustworthiness are so central to the nature of the pathologist's work.... that no pathologist can truly be effective without holding deeply such values" (Harris,2018). The patient centered mind set of physicians in terms of monetary benefits is associated with altruistic behavior. This was confirmed by a study where medical students who were less altruistic chose higher paying medical specialties and those who were more altruistic chose low paying medical specialties. However, other studies suggest that monetary incentives or rewards can always enhance the commitment of pathologists to practice in the interest of patients by promoting higher level of prosocial behavior which is a mixture of both intrinsic and extrinsic incentives. (Lee et. al, 2019). A study conducted by Tammaro shows that, patients interest and well-being is considered one of the core pillars of physician-patient relationship which is threatened by the advancements in medical technology (Tammaro, 2018). Patient centered mind set of physicians might include, for example, continuing to work or providing informal medical advice outside contracted hours, giving free treatment to poor patients in fee for service healthcare systems, and a general willingness to go the extra mile in professional activities. There is much evidence that many doctors and pathologists work beyond their contracted hours, but there is also a growing feeling that patient centered mindset in medicine is declining (Jones, 2002). Technological advances including artificial intelligence are increasingly impacting bedside care in hospitals besides the many advantages it poses and is expected to threaten the physician-patient relationship by reducing empathy and compassion between them (Tammaro, 2018). Patient centered mindset and its importance in maintaining the professional 
autonomy of pathologists and how it could be impacted by AI tools implementation is explored in this study.

\section{Self-Regulation by Pathologists and Validation of AI tools}

Self- regulation is widely regarded as a key element of professional autonomy in general and also is one of the major requirements of the pathology profession for pathologists to possess professional autonomy. Self-regulation was originally instituted as medicine was considered a body of knowledge not easily recognized by ordinary public and external regulation was not seen as feasible because the non-experts will not be able to speak in the same language as the medical professionals (Collier, 2012). However, in today's world of technology, privacy breach, scandals and burn out of medical professionals, it is becoming increasingly difficult for pathologists to put in extra efforts to monitor the performance of their peers for the purpose of increasing the quality of work performed by them as it is a time-consuming activity with little or no direct benefit to patients. Also, earlier medical profession was considered deeply influenced and connected to altruism and integrity which is clearly seen to be deteriorating now. Another issue that has been highlighted in many studies is that pathologists carry out activities by being a part of an organization or a group and constantly coordinate with peers and specialist pathologists for secondary opinion. Therefore, pathologists are seen to be protective of their fellow pathologists (Collier, 2012).

A key component of self-regulation when any new technology is introduced requires validation of new tools or tests. Comparison of any digital pathology technique will need to be verified against the performance of human pathologists with their inherent irreproducibility and day-to-day performance variation. Measures of laboratory and clinical validation should be established for any new tool against a gold standard set by the laboratory professionals. Colling et. al 2019, 
suggests that for pathologists to be confident in the output of algorithms before signing out the main report would need to put the AI tools through validation and verification. There is a need for pathologists to build trust in AI system which at present seems like a black box and this is putting a brake on the speed of AI implementation in digital pathology (Colling et. al, 2019).

This study explores the perception of pathologists on how AI tool implementation could impact their professional autonomy and what should be the approach to validate these tools to increase the trust of patients and physicians on the results given out by pathologists using AI tools.

Artificial Intelligence is paving its way in every field of study, industry and organization faster than any other technological development ever has. One of the major impacts of it is perceived to be in health care industry including professional practice of medical professionals and patient care and safety. The goal of this study was to better understand the outlook of pathologists about the impact of AI tools on their professional autonomy and based on this, their commitment to implement and integrate AI tools in their daily practice. Such an insight would allow more informed steps that should be taken at the level of regulatory compliance, ethical concerns, technical skills and rationale investments before implementing AI tools in pathology practice.

\section{Methodology}

The study was designed to explore the perception of pathologists about the impact of AI on different dimensions of professional autonomy. Semi- structured interview guide comprising of 25 questions was designed (Appendix, Box 1) from a bibliographic search so that major dimensions of professional autonomy would be discussed, like ethical code of conduct, knowledge and skills, self-regulation, patient centered mindset of pathologists. After a few introductory remarks, the interviews were conducted according to the guide. The questions were open-ended, allowing our 
respondents to develop their ideas, or even to digress and venture into subjects not always directly related to the initial question. The participants were free to express themselves. Depending on their interest, involvement in research related to AI and understanding of AI tools, some aspects were more developed than others.

For the data collection process, semi- structured interview guide was designed using the four dimensions of professional autonomy, but we were also open to emerging themes that do not fit within the four dimensions.

Prior to data collection, permission was sought from Research ethics board of Ryerson in accordance to the TCP-2 policy guidelines. It took about 5 weeks from the time research proposal was submitted to REB, revisions were done and final permission to carry out the research was granted. The data collection was done in Toronto, Canada but the pathologists interviewed were from other provinces as well based on their interest and availability. The targeted number of pathologists to be recruited and interviewed were approximately 15-20. Out of which, 8 were successfully interviewed keeping in mind the time constraints of the study, their availability, interest and willingness to interview.

Firstly, 20 pathologists who are currently practicing were identified based on their interest in AI through information posted on their organization website. They were contacted by email to schedule an appointment to discuss the subject of how AI is perceived by them to impact their professional autonomy. Those that did not answer the first time were sent a reminder. 8 pathologists were recruited successfully and interviewed. The interviews took place mainly faceto-face at the participants' workplace or, if this was not possible, they were conducted by telephone. Indeed, pathologists were interviewed by telephone if they were outside Toronto at the 
time of the interview or if they were reluctant to be interviewed face-to-face, mainly because of their schedule.

The interviews were recorded on Otter, with the agreement of the participants, and the corresponding interviews re-transcribed. Then, the transcribed interviews were analyzed using NVIVO software respecting the anonymity of the participants.

The data were analyzed on NVIVO using three stages of the coding process, according to the methodology described in various studies (Houghton et. al, 2017, Maher et. al, 2018). Initial (or open) coding, intermediate (or axial) coding, and advanced (or selective) coding was followed. In the initial coding stage, raw data were produced and labels (codes) attached to them. The raw data comprised of transcribed interview text on the four dimensions of professional autonomy as follows- ethical code of conduct, advanced knowledge and skills, self-regulation, patient centered mindset, In the intermediate coding stage, important text paragraphs were chosen as significant codes and were tagged as nodes and assembled to form categories. In the advanced coding stage, categories were developed from the nodes and four core categories indicative of four dimensions of PA were selected. Finally, a theory was developed that established the links between the categories (Maher et. al, 2018).

\section{Results}

In this section I report the impact of AI on different aspects of professional autonomy of pathologists when/if it is implemented in their daily professional activities.

During the interviews, the several themes which were related to the four constructs of professional autonomy of pathologists- Ethical code of conduct, knowledge and skills, self-regulation, patient 
centered practice of pathology, directly related to autonomy of pathologists. In addition to the usual elements of professional autonomy, the interview text also highlighted some major emerging themes referring to another construct "Validation of AI tools". I will be discussing the direct results obtained from the text analysis for each construct in sequence.

\section{Ethical code of conduct}

Pathologists perform professional activities and they serve as an entity of ethical autonomy by providing disciplinary punishments for misconducts as needed. They are required to carry out these social and other useful activities to get recognized as professionals. To achieve this autonomy, pathologists must adhere to basic ethical framework that includes respecting rights and privacy of patients when dealing with their diagnostic cases.

Several major concerns on the impact of using AI tools on the ethical code of conduct of pathologists have been pointed out by them that directly or majorly corelate to their professional autonomy.

First, many of our respondents have mentioned in the interview that if the AI driven decisions are accepted by pathologists without validation or if the AI driven results are based on certain parameters which is outside the human eyes capability to visualize or understand, it will be the major ethical concern. One of the pathologists illustrated this with an example:

"If AI tools says 99\% about something, I didn't think at all or trust it. I'm going to worry that something's wrong.... But when we're talking about something that AI sees that is not visible to human eye. That's where I worry about the hack, especially if that thing influences accrual of patients to a drug”.

Another respondent commented 
"And the area of rejection or ethical concern is when pathologists accept diagnosis that they can do without proper validation of AI tools and without proper knowledge of it".

Second ethical concern pointed out by majority of pathologists is the issue of equity to access AI tools by organizations or hospitals. One of the pathologists said

"AI is available but has not been implemented at the lab level or the provincial level over all Canada. And that's a huge ethical challenge".

This refers to financial stability of the labs and the availability of expert professionals trained to deliver care by using AI tools.

"Now can we accept that our philosophy and our health care system has always been a single level of care. but AI may increase, shall we say the challenges we have in making that promise, about a single level of care. If it comes up with algorithms that we cannot afford".

Privacy issues are a key ethical concern, mentioned by almost all our respondents. Pathologists worry that patient information could be sold, hacked or leaked from AI solution providers and used for unethical purposes. One pathologist illustrated it like this:

"here is like a million and a half molecular results. Someone would hack (them) and find all the people who have a Ross mutation that is marketable. (They would) create a list, sell it to a third party and suddenly, all those patients are getting flyers: "is your doctor keeping (you up to date about...)?"

However, respondents also pointed out that privacy problems are not new or specific to AI and are primarily a solvable technical problem and it should be the responsibility of the organization and not of the pathologists. 
Another concern pointed out was on using AI tools retroactively. One of the pathologists commented that

"Oh you called something $x$ a month later I sent you something very similar and you called it something different. And, in theory......... we would never find something new .........we would never look back and start changing old reports........ First of all, it's not clearly ethical”.

Another comment added by him was

"Another reason to not do it is some of those patients might not want to be contacted ....... What are you telling me you're telling me something new about my tumor from 10 years ago......some have passed away...... the families don't want to hear anything new or maybe they do..... maybe this will help....my family because there's a genetic syndrome ...so we don't look back and change things".

These comments clearly indicate that it is against the ethical norm of pathologists to evaluate the reports of the past with the latest technology developed whereas AI tools might expose their reports to be evaluated retroactively and thus induce a sense of lifetime discomfort for them and might raise questions on their skill set.

The ethical concern is that the cases of the past and relative patients would have died or been cured by them and their families might not like to know about what they could do to save their loved ones. Also, the ones already cured will not be much interested unless they want to see the trend for their future generations to follow.

Almost all our respondents have discussed the implementation of AI tools to be helpful in providing secondary assistance to pathologists but refrain from accepting that it can ever take the same place as of pathologists. Few comments made by pathologists on this are "And I do accept 
that I make mistakes, because all pathologists make mistakes. And I do accept that if it if the computer thinks I'm wrong. And if I believe that there's some credence to that, then maybe I am wrong, it might cost me to further investigate. It might lead me down that path. It's not that I'm stubbornly going to disagree with a computer once I've made my diagnosis”

"AI wouldn't help very much in terms of making the diagnosis per se but it will help in kind of help our work more efficient but not so much until we're making the final diagnosis"

They said that it might just increase the efficiency of pathologists as it prompts them to get another opinion if the results do not match.

One of the respondents commented that

" Pathologists would be conservative in taking the opinion of AI tools".

One of the pathologists says that: "So if my test is calibrated to, to, to detect acne or a Bunyan on your foot. I'm not worried about false positives, as much as if it's detecting pancreatic cancer"

Pathologists autonomous behavior might defer depending upon the severity of the cases. If cases are not chronic, they might completely rely on the results of AI tools versus if the cases are critical, they might give in more time to go through the results of AI tools

Third theme that emerge is that validating these tools is also important to protect the pathologists from being accountable in the future for the results of the past. One of the pathologists discussed this

" 10 years from now, there might be a better AI program that find something in the exact same slider digital image that we didn't see before, but by definition, if we had an AI system helping us out and that was validated in sanctions by some sort of regulatory body, we will be like, I don't really care what your AI program shows 10 years later. This is the program that we used at that time” 


\section{Knowledge and skills of Pathologists}

Knowledge and skills of pathologists are important in pathology practice and has developed over the course of time with the help of research and technological development. Continuous development of their knowledge and skills with the new emerging trends of innovation and research is very important to maintain their professional autonomy. One of our pathologists has put it in this way

" With time pathology has developed from slides made of wood to modern pathology where we have glass slides now".

Several major themes emerge on this aspect of professional autonomy. Pathologists anticipate that before the AI tools are integrated in their daily work, they need to learn the technical skills related to these tools.

Some of them comment

", first, you have to be able to use it or need a certain level of computer competency, how to use this program, how to make it work. And when it has problem, how to solve it, it's just some basic skill. Like, I want to imagine every artificial intelligence system will have some intrinsic problem and is not 100\% approved.................. if pathologist can't, update their skills in terms of getting know and understand how an artificial intelligence system work., eventually, we want to be competent for this job.

"I think that probably there need to be some curriculum of AI, just like we do for molecular pathology.........royal pathology exam, there are questions on the molecular biology of the cancer, ......with AI, and they already like right now is still not mandatory part of the curriculum because it is not ready to be used in clinical setting...........then definitely you need to have mandatory training of AI............AI in pathologists certification””. 
AI tools if not validated cannot be used independently to give diagnosis and thus requires the pathologists to gain more knowledge of the software, algorithms and its analysis.

"AI tools will change our profession to be more of a verifier than a screener".

They mention at many different instances that AI tools can be used for the repetitive tasks that takes up a lot of time and attention of pathologists like screening and flagging the slides. This would save on their time but at the same time it would change their profession to more of a verifier than screener. Physicians and patients might have more trust on the reports screened and verified by pathologists and AI tools both as it would give them an edge of surety that the results have been checked twice.

\section{Self-regulation and Validation of AI tools}

Some of the themes that originate from the interview text is the regulation of pathologists by regulatory bodies and peers. Pathologists perform professional activities by setting up regulations for their co-workers or members in the organization. They are required to carry out self-regulatory activities to be recognized as professionals as a part of their autonomy. Some of the different activities performed by pathologists to monitor themselves and their peer's performance include peer reviewing and sending the report to specialists for second opinion. Most of the factors that are monitored to review their performance are not favored by pathologists due to reliability issues or because it consumes a lot of time and efforts of already overworked pathologists without any cost benefit. One of the pathologists mentioned that

"so, it will be much better off to spend our resources in another way, other than doing the reviews". 
In support of this, one of our pathologists mentions that due to the extra cost and more work, time and use of resources associated with the process of regulation, some private labs have stopped the process of quality assurance of pathologists.

He said "very recently the College of Physicians and Surgeons did audits so they would send somebody out and then this fellow come in and say show me your last 50 cases with these diagnoses. Okay. They found that return was so very little they just recently stopped with me”.

Professional autonomy of pathologists and QA go hand in hand as it strengthens the trust of patients on the independent practice of pathologists. But the manual process of peer reviewing is a lot of work according to some of our respondents. Some of the private pathology labs have even stopped the manual processes of quality assurance.

Some major themes have emerged exhibiting the impact of using AI tools on the regulatory processes of the pathologists. According to interviews, pathologists' comment that these tools are anticipated to flag the results outside the correct parameters and thus would speed up the review process done by pathologists, saving on their time and resources as well.

"AI can help me speed up the process of reviewing cases that I get from offsite. Ai would highlight slides having cancer so.... I will be happy, or at least it can help me speed up the process versus like yeah......slide has cancer, so I only need to focus on those slides"

Respondents feel they could then give more time to the cases that need attention and thus build up on their efficiency. Pathologists do not mind being reviewed by AI tools as an alert mechanism to give more time, attention and resources where needed and feel that these tools would be of great efficiency if used to flag mistakes of pathologists before the reports are sent out to minimize the negative impact on patients. 
On a negative aspect, pathologists have mentioned that human pathologists while reviewing cases keep many variables related to the patients case history and present in context which is outside the capability of AI tools and thus these can never be used independently to monitor the pathologists. Pathologists have referred AI tools as a big brother, a third eye or a resident to keep track of pathologist's work or just to watch over their actions.

However, another respondent quoted that

"Let's start with the residents, what would make me look at less or review the residents less. You would have to take the legal responsibility, away from me for what they missed. And it would be the same with AI, right".

Based on pathologists comment, if they are not held legally responsible, they would review the cases less carefully and responsibly and they feel that if AI tools are implemented and used for reviewing or diagnosing, the legal responsibility might get taken away from them.

Another threat to the autonomous judgement of pathologist might arise if the physicians try to form their own opinion of pathology cases independently or use it to identify the best tests to be ordered instead of seeking advice from pathologists. In such instances, pathologists should not be held responsible for misdiagnosis.

One of the pathologists said "AI might change that Physicians might learn through the AI on their own. Something that they can't learn from pathologists............... opinion independent of the pathology and then there might be more discussion because of AI... it might bridge the gap between clinicians and the biologists". 
Self-regulation traditionally implies that only pathologists can assess the validity of expertise in pathology, but that AI may allow non-pathologists to come up with counter-expertise without getting the opinion of a pathologist.

\section{Self-regulation- Validation of AI tools}

One of the important pre-requisites of using AI tools as a performance grader of pathologists is the need of validating these tools.

Two themes emerged referring to the impact of AI on the need to get these tools validated. One of these themes are the need of validating AI tools to be trusted by patients, public, physicians, surgeons and pathologists. It has been compared with the molecular chemistry kits that are standardized before conducting the actual test. One of the pathologists mention that

"But when we buy molecular kits, we still validate it. And if we run out of it or If it expires then we get a new kit. We sometimes validate again even though we have validated it before, we make sure it works first. So, the, the burden of making sure something works for us is still there".

Second theme that emerges prominently refers to the stakeholders that should be involved in the validation of these tools. Ideally, according to our respondents, laboratory pathologists and professionals must be involved in all the steps of the validation as they would have more knowledge of how to validate or install these software's than the physicians or administrators. However, this is sometimes not appreciated by physicians, researchers or administrators. One of the pathologists points out that

"in all those steps of validation, I think the lab, the lab professionals should get involved because it's certainly the skill set for the lab professionals or pathologists to solve this problem. This isn't always appreciated, either by administrators or clinicians or researchers" 
These comments refer to the perception of pathologists on the importance of validating AI tools and who should validate them before they are integrated in the daily activities of pathologists and how AI tools if validated would be trusted and accepted more by patients.

One of the respondents mention that

"If for example. the AI tools get validated still AI would use pathologist as gold standard and at the end of the day cases are challenging and even extra pathologists may not be able to get to the diagnosis ................... if AI is used in this case then it can never surpass a pathologist diagnosis

\section{Patient centered mindset of pathologists}

One of the major aspects of autonomous pathology practice is to have a patient centered mindset before putting their financial or personal interests. Due to this, it is always required by the pathologists to follow set standard rules while setting up treatment plans or sending out diagnostic reports in the best interest of patients. Pathologists take appropriate time to analyze and interpret the cases before sending out reports and avoid rushing for any reason. They consider many factors from the past and present of patients that might in some way impact the reports.

Some of the themes from the interview text that are prominently related to patient centered pathology practice are illustrated below.

Pathologists practice the role of gatekeeper of resources as part of their professional autonomy keeping in mind the benefit of patients and allocating resources in their best interest. They are the most conservative when it comes to ordering tests compared to the physicians. Pathologists have highlighted that there are constant battles between pathologists and clinicians about which tests are necessary to be done or not and pathologists are able to get their way out only rarely. One of the pathologists have stated that 
" we are stewards of the system and have to be mindful of the resources and time spent on tests that are not needed and exposing patients to unnecessary radiations which might lead to devastating outcomes".

This was favored by other pathologists as well by stating that pathologists never get swayed by the unreasonable demands of the patients and remain focused on what tests are to be ordered for making the appropriate decisions otherwise it would lead to wastage of resources and time.

According to our respondents, pathologists are involved in repetitive tasks and AI tools would make them more focused on accuracy and efficiency of results. They talk about the practice of pathology being more or less subjective and how AI tools could make pathologists more objective. One of the comments made by them are:

Well there is a lot of repetitive work in pathology, like counting cells and grading tumour, more repetitive, and there are also observed differences, like from one side to the other it is not $100 \%$ objective. Okay. So, in that perspective, I think artificial intelligence will help a lot to make our evaluation more consistent in terms of annotation. So, I went back and actually these tools will help more, to make results more consistent.

However, if AI tools are implemented and government funding is not provided to organizations for using these tools equally for every case, it might lead to pathologists being reimbursed per slide screened by AI tools. This might turn pathologists into becoming wasteful. Pathologists might then focus on using these AI tools for every case to confirm the efficiency and accuracy of results and care less for the wasting resources.

One of the pathologist states that "unless the government willing to compensate for that it actually will negatively impact our salary. We don't get paid to do to look at more cases. So, it's not that 
government will pay us more if we use AI, we can solve $50 \%$ more cases, but the cost of AI tools will not come from my pocket, the government will have to compensate the bill".

About 6 pathologists have mentioned that AI tools will lack the human element as these are tools and when implemented will be more focused on efficiency and accuracy.

For example, So, for AI is all about making the right diagnosis for pathology, and the future I would say, a certain amount of time will have to be spend talking to the medical team who was incharge of the management. And, you know, after each discussion, the final management may not be the same as the AI suggests, because of real issues.

Because of the efficiency enhancement by AI tools, government might declare incentives for pathologists to use AI tools, this would completely change the dynamics of using tools more for efficiency and less for patients' interest. if the government fails to introduce incentives, the use of AI tools might affect their salary. Another solution to this problem discussed by our respondents is that AI tools must be programmed in a way that it uses the existing resources and we do not need to change the complete infrastructure for it. It should be programmed in a way that once it is implemented in the organization, it can be used for all the cases coming to that organization without charging the patients or hospitals.

One of the respondents says: "AI is going to have to work in the existing resource environment. Yeah, I guess we can back up and say well are you going to program your AI, so it works within the existing resources"

Pathologists do not practice in isolation and final diagnostic report is released with their sign out after complete surety and in some critical cases, second opinion from peers is taken before releasing the final report 
Another theme referring to this aspect is that pathologists protect their professional autonomy by avoiding staying in contact with the patients. Patients have complete right on their treatments but often have vague knowledge from online sources. Almost all the pathologist's reason that they do not want their decision to be swayed because of emotional breakdown.

One of the comments go like: pathology is objective. So, I stay away from patients, because I don't want to be swayed by how nice emotions. And I made you know, I'm an emotional being

despite my outward appearance. And So, as a result, I can be swayed one way or the other and we swayed each other the pathologist's do it...we indirectly we do that. And it's hard to be objective in that sense.

Sometimes, pathologists have no communication with patients and its only through the samples sent for repeating tests they anticipate that the patient is still under study or is alive. If AI tools are implemented, it might be possible that patients could try to use these tools on their digital slides available to them and come to an anticipated diagnosis which might create conflict between the final reports sent out by pathologists.

\section{Discussion and Practical Implications}

Professional control of pathologists on diagnostic decision making for physicians and patients is now changing with AI tools developing at a rapid pace than any other technology till date. Professional autonomy is one of the most important attributes held by pathologists which makes them more responsible, patient centered, ethically sound and mindful of the quality of their performance while giving independent diagnosis on cases. In the era of technological advances, AI is now reaching the field of pathology and in order to have a competitive edge, pathologist must be prepared to face the arrival of AI tools. Besides the never-ending discussion of AI in healthcare, 
we also found in this study that in accordance to the perception of pathologists, diverse themes have emerged among each dimension of professional autonomy.

We discuss the different aspects of professional autonomy and other potential factors that might also be impacted by AI tools. The dimensions of professional autonomy are 1) Ethical code of conduct 2) Advanced knowledge and skills, 3) Self-regulation and validation of AI tools, and 4) Patient centered mindset. We discuss them successively

1) In terms of ethical code of conduct, from the results indicating perception of pathologists there is a potential apprehension among the respondents that the use of AI tools might interfere with respecting rights and privacy of patients. Pathologists lack proper technical skills to evaluate the results given by AI tools that might come up as a major limitation on their part to assess if the tool or software is tweaked or hacked by any organization eventually impacting privacy of patients. Pathologists have been making diagnostic decisions autonomously since ages by staining slides and screening them under the microscope without the use of any kind of tools. However, with the introduction of AI tools, the cases reported by pathologists in the past could be unfairly monitored today and this is perceived as stressful by them and ethically unfair because these tools were not developed to be used earlier. Pathologists independent practice of sending out reports autonomously might force them to use these tools to navigate their way out with physicians on diagnostic decisions which is yet again against their ethical practice. Professional autonomy practiced by pathologists makes them more responsible towards their diagnostic decisions but if AI tools are used to assist the ordinary pathologist, it might bias there decisions by highlighting the slides that according to AI tools need more attention and pathologists would end up concentrating more on the cases labelled as critical by these tools and not by pathologists themselves. This clearly is an example of putting the pathologists out of the autonomous process 
of decision making and could be a threat to their professional autonomy by making them listen to AI tools at the granular level, thus causing harm to patient safety and increasing the rights of AI taken as an autonomous tool by the pathologists for most of their diagnostic cases.

This is consistent with the study done by Rigby (Rigby, 2019), Blease (Blease et. al, 2019) and Allen, where the authors have demonstrated that patient rights, privacy and proper governance as one of the major ethical concerns that would bring about a transition to the role of pathologists from being autonomous or being "in the loop" when it comes to diagnostic decisions to their role where AI tools become autonomous and keeps the pathologists "out of the loop"( Allen, 2019). A study recently conducted by Keskinbora, talks in detail about the importance of ethics in terms of criteria of validation of AI tools, equity, patient privacy and data hacking issues (Keskinbora, 2019). Whereas this current study also highlights a new ethical concern, the unfair use of AI tools retroactively on past decisions of pathologists. It is ethically unfair to use AI tools for cases that were diagnosed by pathologists microscopically because the patients corresponding to those cases have been cured or are not alive now and their families most probably will not be interested in finding out what they missed years ago since these tools were not developed. The only reason these tools could be preferred by patients to be used retroactively is to make their families aware of what to expect from generations to come or for the current generation. However, it would still not be fair on pathologist's part to use these tools retroactively for monitoring their diagnostic decisive performance and unfair on the patient's part for making them feel unfortunate. Based on this, it would be stressful for pathologists as they would always stay in distress while making diagnostic decisions and will no longer enjoy professional autonomy completely.

Based on the results, certain regulatory guidelines must be established that lays down the criteria under which AI tools could be used on cases from the past to prevent the unfair retroactive 
monitoring of cases diagnosed by pathologists. Consideration of these ethical issues is very important before implementing AI tools for two major reasons 1. Patient safety- In situations when diagnostic results driven by $\mathrm{AI}$ might be wrong and pathologists still move forward with the diagnosis due to lack of technical skills to understand the software, 2. Patient data might be available for everyone to play around with it or if it gets hacked and tweaked resulting in uncontrolled emails being sent to patients advising them about the different treatment options and doctors.

2) Advanced Knowledge and skills: According to our respondents, implementation of AI tools will augment pathologist's role to be more of a verifier than screener and pathologists will have to adapt to the technical changes these tools would bring, by getting hands on training of AI tools. However, they also perceive that over the course of time this would make them lose their expertise of traditional screening of slides under the microscope. Pathologists would come out to be different kind of professionals who are more trained as verifiers and interpreters and less as screeners. Therefore, it is agreed upon by respondents that it is hard for an ordinary pathologist to learn the skills with the same speed as the technical advancements with respect to AI to attain an equilibrium between the two. If equilibrium is not attained, it is expected by the respondents that pathology practice might age resulting in decreasing reliance of patients or physicians on the reports sent out by a usual pathologist. They would in some way want AI tools or software to check over the diagnosis made by pathologists as they would want the latest technology benefits. This would be taken as a threat to the professional independent judgement making power of pathologists as they would increasingly start depending on the results given by the AI tools and in seeking expertise in technical skills to understand the process of using these tools. A pathologist who performs the daily practice of screening and verification of glass slides under the microscope and then sends out 
the diagnostic report of his best independent clinical judgement might then be seen investing more time and effort in learning the computer language and skills of AI tools rather than investing in the pathology practice

One of the reviews compiled by Salla and co- authors suggests the loss of diagnostic and cognitive skills of physicians in the long run ones AI tools are integrated in daily practice (Salla et. al, 2018). In another comprehensive literature survey, Ahuja argues that AI tools will outperform the screening abilities of pathologists by providing rapid analysis of large amount of image data with same accuracy and efficiency as compared to human pathologist and thus is expected to improve the practice of pathology by saving on time. AI is likely to support and augment pathologists by taking away the routine parts of their work like screening and hopefully enabling the pathologists to spend more precious time on other more important tasks. (Ahuja, 2019). This supports our finding that AI tools might bring about pathologists as more of verifiers by doing most of the screening work more efficiently and in less time than pathologists. A very recent study talks about the exponential growth in the application of AI tools in pathology and states that PubMed shows an almost exponential growth in publications in pathology AI on the last 5 years. Unfortunately, as is typical, this has not yet translated into a similar growth in diagnostic practice and the translation of research to clinical diagnostics (Serag et.al, 2019). Our results however suggest that AI tools might change an ordinary pathologist to be a different kind of professional having more technical skills and expertise and investing more time in verifying the cases screened by AI tools as critical and leaving the benign cases behind but the decision making of pathologists would then be more based on the decision given by AI tools at the primary level. Patients or physicians might not be confident if the case was considered benign by an AI tool and the pathologists would never give a second thought to it. Also, if the technical skills of pathologists do not develop with the 
same speed as that of AI tools which is unlikely, pathologists won't be able to judge whether the AI tools have correctly screened all the slides as benign or cancerous. Therefore, AI tools will augment the practice of pathology but is likely to reduce the level of autonomous decision making done by pathologists at the primary level.

Pathologists must be given hands on training of AI tools and technical software starting from there residency curriculum to give them competitive edge and necessary professional growth. Pathologists practicing traditional pathology would also have to learn technical skills in order to compete with the changing technological trends and they can be better in their practice as they would have expertise in both traditional pathology, digital image pathology, screening and interpretation.

3) Self-regulation and validation of AI tools- Our result findings suggest that self-regulation is very important to build upon the trust of patients and physicians on the reports released by an ordinary pathologist and for the pathologists to be less cavalier of the diagnosis done by them while enjoying professional autonomy. However, as results show that second opinions increase the workload and requires a lot of time and resources to be put in by an ordinary pathologist with no cost benefit at all but cannot be over-looked or omitted out as it keeps a check on the quality of diagnostic reports sent out by pathologists. For pathologists are professionals and their practice are licensed by governing body for the knowledge and expertise they hold and thus they are given a privilege to self-govern themselves to hold rights and responsibility of giving diagnosis autonomously. This is consistent with the review reported by Collier that talked about the importance of self-regulation to maintain the autonomy of medical professionals and that it is perceived as a demanding and expensive process by them (Collier, 2012). Pathologists favor the use of AI tools as these can flag the results or reports that need more attention of pathologists 
compared to the benign cases. The responses suggest that AI tools could challenge the selfregulation by allowing third party to watch over the performance of pathologists and highlight the cases that needs more attention before the results are sent out and can improve the efficiency as well as performance of pathologists but at the same time, a part of their autonomy would be taken up by these tools. Professional autonomy practiced by pathologists makes them more responsible towards patients and physicians which is also because their practice is self-regulated and monitored by the regulatory bodies. If AI tools are used to monitor their performance, it might increase their efficiency but a part of their autonomy could be taken up by these tools as they would be in constant distress of their performance being monitored and impact their professional development. Also, if pathologists are being monitored by an external tool like AI, an ordinary pathologist would rely more on the performance review done by these tools and pathologists might lose the confidence to review the performance of their peers as AI tools would give counter expertise to non- pathologists thus impacting self-regulation as an important dimension of professional autonomy.

Walter and Lopez also reported similar findings that IT tools work by coding information and storing in the form of data that can be shared between professionals and co-workers thus sharing the knowledge, skills and expertise with non-professionals (Walter and Lopez 2008).

One of the major key ideas retrieved from the interviews of my respondents is validation of AI tools. It is considered very important by our respondents for the process of successful implementation and integration of AI tools in their daily practice. Once these tools are validated, the diagnosis done by them will be much more readily accepted and cannot be questioned over the course of time. However, according to our respondents, these tools must be validated by pathologists, but these validated tools would still use pathologist's diagnosis as gold standard as mentioned in results. Validation of these tools has been noticed as a major concern among 
respondents and can be considered the central locus of professional autonomy as whoever holds the legitimacy of the tools from among the human pathologists and lab professionals or AI software may control the legitimacy of the profession as well. Many of our respondents are of the opinion that pathologists must be involved in the validation of these tools as they are most aware of the dynamics of pathology cases and diagnostic details associated with it. The involvement of pathologists in validating these tools is important for the results to be accurate and efficient. One of the reports released by CPA, Ontario talks about AI if trained with labelled data that is having conscious or unconscious bias related to human traits for example, Sexism or ageism or racism then its processing of information or diagnosis will be distorted (CPA,Ontario, 2019).

A recent study by Panch et. al and Cath conducted on AI governance reported similar findings on the importance of implementing AI tools to their maximum potential by validating them. According to these studies, to realize the potential of AI across health systems, more fundamental issues related to the availability of health data, its ownership and its use are some of the factors that must be addressed for validating these tools (Panch et. al, 2019). According to our respondents, pathologists must be involved in the validation of AI tools as they would be the owner of patient's diagnostic data and most aware and familiar with it. Moreover, pathologists would be able to validate these tools with least biases as the central objective of pathology practice is to hold patient centered mindset and to work for the wellbeing inclined towards patients' interest first. Pathologists are legally responsible for the diagnostic reports released by their sign out and would be held accountable for any errors that arise irrespective of the tools used or not and thus should represent legitimate power to assign gold standard of validation to these tools for accurate results. Public discourse and policy interventions are needed to design the framework of validation. 
However, in private sector, the companies able to gather large amounts of data are the ones that would be involved in the validation of these tools (Cath, 2018).

Therefore, public policies or interventions must be designed by considering the criteria of involving the pathologists when it comes to validating AI tools for giving diagnosis on slides. However, they also feel that if validated AI tools are used to review their performance retroactively then it will pose a significant threat to their professional development as their performance could be tracked by just means of a click. AI tools are perceived by pathologists as a threat to their professional autonomy by giving counter expertise to non-pathologists and thus deteriorating the trust of physicians and patients by making them rely more on the results reviewed by AI tools.

There is a huge possibility that non pathologists would try to screen the images with these AI tools without having any knowledge of the field of pathology unless these tools are validated.

4) Patient centered mindset: Our respondents consider themselves gatekeepers of resources which makes them strive for effectiveness and the best interest of patients before sending out diagnostic reports or before ordering diagnostic tests. Pathologists practice autonomously by being more mindful of the diagnostic choices they make and always have a patient centered mindset while doing so. These pathologists prefer to not stay in direct contact with the patients to prevent getting swayed by unreasonable demands of tests as one aspect of their autonomy is also to protect patients from unnecessary radiations that might cause devastating results. Besides the dynamics of patient pathologist relationship, pathologists also try their best to navigate their way out with the physicians for ordering the tests or tools that are necessary for the diagnosis.

If AI tools are implemented, it would require complete digitization of the infrastructure and appropriate government funding equally distributed across all the organizations and hospitals. Pathologists are salaried and not fee for service and they are concerned about their salaries being 
impacted due to the use of AI tools if the government does not give funding for the establishment and implementation of AI tools, equally to all the organizations and hospitals. Respondents feel that if appropriate government funding is not provided, pathologists might have to get reimbursed for using AI per case. This might change pathologists into being wasteful as they might use AI tools for every slide to improve the accuracy of the results and be less mindful of the wasting resources. In addition to this, in the light of our results, AI tools will cover some of the repetitive tasks for pathologists that are considered laborious and thus would give them more time to concentrate on result accuracy and interpretation. This might make pathologists more inclined to use these tools for not missing out on the accuracy and efficiency check. In other words, it will make the pathologists more objective or focused on accurate results. This on one hand would improve the treatment decisions but on the other hand, it might make the pathologists wasteful towards resources or take away the gate keeper role of pathologists by making them want to use the tools for less important cases too. Pathologists also feel that AI tools might make the diagnostic cases more transparent to patients and encourage them to start demanding the use of these tools for every case, thus impacting the gatekeeper role of pathologists. According to a recent study, in some of the other parts of the world, AI tools have started to slip out of the doctors' hands to be used directly by individuals, although physicians consider the performance of these tools in terms of clinical evaluation is not yet sufficient to ensure confidence in patient safety (Lai et.al, 2020).

According to respondents, implementation of AI tools might make pathologists more conscious of the professional autonomy they hold in terms of making autonomous decisions related to types of tests that are needed or if at all AI tools needs to be used per case because patients and physicians would have equal knowledge of diagnostic tests needed for respective cases and they might start demanding the use of AI tools per case. This might threaten the role of pathologists as stewards of 
the system. According to our result findings, pathologists would need to coordinate, collaborate and communicate more with non-pathologists including physicians and other co-workers and become more involved in the patient centered network. One of the latest studies talks about the future of laboratory professions in times of innovation and advancing technology that pathologists will have to be a part of patient centered networks and will have to collaborate more with other stakeholders and patients (Plebani et. al, 2019).

Practically government must take initiative to provide funding and incentives for these tools to be implemented equally in all organizations and hospitals should not charge for each case that is being assisted by these tools for efficient diagnosis. A universal AI system across provinces must be established for pathologists to practice with a patient centered mindset and not waste time and resources using these tools separately for each case. This will allow pathologists salaries not to be impacted and the mode of payment will not be altered along with patients receiving universal patient care. Policy design and intervention should emphasize more on engaging pathologists in patients and physicians' network for sharing and exchanging knowledge for better sustainable trust and belief on their pathology practice even if they are using AI tools for decision making assistance. Canadian health system must include these parameters to measure and allocate the resources efficiently and accommodate the rise in cost and expenditure that might occur with AI tools for successful integration and implementation of these tools.

\section{Future research:}

Future research must focus on factors that are preventing to fill in this knowledge gap and factors that are slowing down the integration and implementation of Artificial Intelligent tools in the daily activities of pathologists. Maybe it is fear of change or lack of computer skills that are perceived by pathologists as convoluted. This research study highlighted some very important points related 
to addition of hands on training of AI tools in the residency curriculum of pathologists. Future research must be done to explore the different ways the curriculum can be designed to provide best benefits to pathology residents. Also, research focused on regulatory guidelines considering ethical issues related to equity, conscious or sub conscious bias and data privacy concern is important for patient safety. Further research is important to lay down factors or criteria on which AI tools will be allowed to fairly review the cases retroactively, maybe to see the diagnostic trends that run in a family for tracking genetic disorders.

During the data collection process midway, based on the answers given by respondents, one additional factor "validation of AI tools" was found important to be explored specifically in more detail. At this point about 4 respondents were already interviewed and were not asked questions to probe the impact of validation of AI tools on their professional autonomy. Further research is needed to interview more respondents to probe the impact of validating these tools and the process of it all

It is also important to study the perception of patients about the stakeholders who should be involved in validating the AI tools. It is vital to direct the researches to map the road for trouble free implementation of AI tools in the daily activities of pathologists for better patient care with safety, efficiency and cost benefit without causing any threat to their professional autonomy.

\section{Limitations}

This study aims to focus on the perception of pathologists on the impact of Artificial Intelligence on four dimensions of professional autonomy. However, some of the limitations this study has must be noted. Firstly, the sample size of 8 respondents is less than what was targeted initially due to the time constraints involving data collection period. Also, the sample size was less due to the 
limited availability of respondents as this study focuses on the perception of one group of medical professionals that are "practicing pathologists" interested in the field of AI.

Secondly, the pathologists that were interviewed had good theoretical knowledge of AI but there was a considerable amount of knowledge gap in technical skills required to work with these tools, which I felt was a drawback in their perception of the impact of these tools.

Third, one of the variables "validation of AI tools" was not explored as much as it should have been due to time constraint.

\section{Conclusion:}

Professional autonomy of pathologists makes them more responsible towards their diagnostic decision making but use of AI tools might impact that in variety of ways. AI tools are entering every industry and are anticipated to make the daily activities of pathologists easier in less time and with more efficiency and accuracy. This study is focused on exploring the perception of pathologists on how these tools could impact the four dimensions of their professional autonomy which are as follows: ethical code of conduct, advanced knowledge and skills, self- regulation and validation of AI tools, and patient centered mindset (Tezuka, 2014). Pathologists are concerned about catching up with the technical skills needed to understand these tools and the results driven out by them to gain competitive edge and emerge as pathologists of a different kind who would be more recognized as verifiers than screeners. It would be ethically unfair and a threat to patient rights and safety if AI tools are used by them without understanding the tools well. Pathologists perceive a certain amount of anxiety with the possibility of being monitored by AI tools retroactively as it would be unfair as well as not very useful for patients except for predicting the genetic trends. Pathologists want to be focused on patient well-being and interest and somewhere 
across the lines they anticipate AI tools to make them more focused on efficiency and accuracy and less on their patient centered role as gate keeper of resources.

This study emphasizes on the above factors to pose as benefits, hurdles or threats of using AI tools on professional autonomy of pathologists which could make it easier to explore reasons that could be delaying or preventing the implementation of AI tools in the daily practice of pathologists. This study would be beneficial to policy makers and advisors to map the road to successful implementation of AI tools in pathology labs, hospitals and health care organizations. 


\section{Appendix A.}

\section{Interview guide}

The focus of my research thesis is to investigate the relationship between AI and professional autonomy, and I want to explore how the different elements of Professional autonomy of pathologists will be impacted by Artificial Intelligence (AI).

AI in pathology can be defined as pattern recognition method incorporating clinical, radiological and genomic data to accurately diagnose diseases and predict patient prognosis.

Some of the opportunities of AI in pathology are the development of algorithms to make disease diagnosis fast and efficient, helping in classifying the disease according to its severity, over all modifying the role of pathologists in medicine practice by increasing their value, efficiency and personal satisfaction.

1. Can you please tell me briefly about your career history and your main activities as pathologist?

2. What does professional autonomy mean to you in terms of your clinical decisions or your clinical judgement as a pathologist?

3. How do you think AI will impact your daily work in the coming years?

Probe: If their comes a situation when your showing what diagnosis your making is correct but it does not match with the results of AI and in the back of your head your constantly thinking what if I am wrong and AI is right. So, you might think if you are not following AI tools you might be wrong. Is it that these tools might induce bias in your decision making?

4. What do you think will be the impact of AI on career opportunities for pathologists?

5. How can AI transform processes in the lab or in pathology?

6. What kind of applications of AI would you readily embrace? What kind of applications would you reject? (be ready to provide examples) Not Mandatory questions.

7. If you were to use AI to support your decisions and it resulted in a medical error being made, to what extent or in what condition should you not be held accountable?

\section{Knowledge and skills}

8. What do you think will be the impact of AI on your skill set, what skill sets will become less or more important or will remain same?

Or

If you were told that tomorrow an AI tool was introduced in your workplace to support you, what would you need to learn?

Probe: What do you mean by risk? Can you elaborate on that?

9. Assuming AI will be implemented, how will that transform the training and credentialing of pathologists in your organization?

10. How do you think the role of technologist or other clinical staff in your organization will or can evolve with the introduction of AI?

11. How do you think the relations of technologist or other clinical staff with pathologists will be impacted? Not Mandatory questions. 


\section{Self-regulation}

12. How is quality of your work currently reviewed?

13. How would you feel about your performance being monitored or compared to an algorithm's conclusions (by an administrator, by a senior pathologist), about AI being used for quality assurance?

\section{Patient Centered Practice}

14. Part of the professional status of the clinician is that you are putting the patients first and that verses your interest of the organization your working for, does that influence your practice?

\section{OR}

15. How do you see AI will impact your ability to put patients interest first?

Probe: Could the gate keeper role become a challenge for instance? The challenge would be whether how much it costs to implement the tools. You might think it is for the benefit of patients, but they would say hey it is not worth it.

\section{Behavior to practice ethically}

16. What ethical challenges could you face using AI?

17. How do you think AI can impact the privacy of patient's clinical data?

Probe: Isn't it something that you feel like personally liable to enforce within your organization. Eg: isn't it the main duty of pathologists to keep that report of patients confidential?

18. A patient has full right in decisional processes related to their health. Do you think AI would generate any effect on this?

Probe: Okay, so it is patient centered, because I think that's great, but still people may feel overconfident specially the youth.

Probe: What if patient demands use of an algorithm for diagnostic decision and you don't want to use it. Isn't that a bit annoying?

19. Patients expect their diagnostic results to be evaluated by reliable pathologists, as they have complete trust on their knowledge, will this trust be impacted by AI.

20. What can be the impact of AI on physician's trust over diagnostic decisions made by you as a pathologist?

\section{OR}

Would the physicians still rely on the results generated by AI compared to pathologists?

Probe: Is it possible that the physician would try to use these algorithms in pathology before referring to pathologist. Eg: A patient asks the physician to contact you and the physician tells him that he can do it himself. 


\section{References}

Ahuja A. S. (2019). The impact of artificial intelligence in medicine on the future role of the physician. PeerJ, 7, e 7702.

AMA J Ethics. 2019;21(2): E121-124.

Allen, Timothy Craig,M.D., J.D. (2019). Regulating artificial intelligence for a successful pathology future. Archives of Pathology \& Laboratory Medicine, 143(10), 1175-1179.

Avasthi, A., Ghosh, A., Sarkar, S., \& Grover, S. (2013). Ethics in medical research: General principles with special reference to psychiatry research. Indian journal of psychiatry, 55(1), 8691.

Bera K, Schalper KA, Rimm DL, Velcheti V, Madabhushi A. Artificial intelligence in digital pathology - new tools for diagnosis and precision oncology. Nat Rev Clin Oncol. 2019;16(11):703715.

Black-Schaffer, W. S., Morrow, J. S., Prystowsky, M. B., \& Steinberg, J. J. (2016). Training Pathology Residents to Practice 21st Century Medicine: A Proposal. Academic pathology, 3, 2374289516665393. https://doi.org/10.1177/2374289516665393

Blease C, Kaptchuk TJ, Bernstein MH, Mandl KD, Halamka JD, DesRoches CM. (2019). Artificial Intelligence and the Future of Primary Care: Exploratory Qualitative Study of UK General Practitioners’ Views. J Med Internet Res ;21(3):e12802.

Camillo Lamanna, MMathPhil, and Lauren Byrne. (2018). Should Artificial Intelligence Augment Medical Decision Making? The Case for an Autonomy Algorithm. AMA Journal of Ethics,20(9). 
Colling, R., Pitman, H., Oien, K., Rajpoot, N., Macklin, P., , Snead, D., Sackville, T. and Verrill, C. (2019), Artificial intelligence in digital pathology: a roadmap to routine use in clinical practice. J. Pathol., 249: 143-150.

CMAJ 2017 November 6; 189:E 1372. doi: 10.1503/cmaj.109-5513.

Cath C. (2018). Governing artificial intelligence: ethical, legal and technical opportunities and challenges. Philosophical transactions. Series A, Mathematical, physical, and engineering sciences, 376(2133), 20180080.

Chang, H. Y., Jung, C. K., Woo, J. I., Lee, S., Cho, J., Kim, S. W., \& Kwak, T. Y. (2019). Artificial Intelligence in Pathology. Journal of pathology and translational medicine, 53(1), 1-12. https://doi.org/10.4132/jptm.2018.12.16

Collier R. (2012). Professionalism: the privilege and burden of self-regulation. CMAJ : Canadian Medical Association journal = journal de l'Association medicale canadienne, 184(14), 1559-1560.

CPA Ontario.(2019). Evolving alongside Artificial Intelligence (PDF File). Retrieved from https://media.cpaontario.ca/insights/ai/cpa-ontario-evolving-alongside-artificial-intelligence.pdf.

Cruess, S. R., Johnston, S., \& Cruess, R. L. (2004). Professionalism for medicine: opportunities and obligations. The Iowa orthopaedic journal, 24, 9-14.

Doctors of BC. (2016). Professional Autonomy. Economics and Policy Analysis Department. Policy Statement.

Emmanuel Letouzé, Alex Pentland. (2018). Towards a Human Artificial Intelligence for Human Development. ITU Journal: ICT Discoveries, Special Issue No. 2. 
Epstein, R. M., \& Street, R. L., Jr (2011). The values and value of patient-centered care. Annals of family medicine, 9(2), 100-103. https://doi.org/10.1370/afm.1239

Esmaeilzadeh, P., \& Sambasivan, M. (2012). Healthcare Professionals’ Adoption of Clinical IT in Hospital: A View of Relationship between Healthcare Professionals and Hospital. Management,2(5), 161-170.

Felgner, S., Ex, P., \& Henschke, C. (2018). Physicians' Decision Making on Adoption of New Technologies and Role of Coverage with Evidence Development: A Qualitative Study. Value in Health,21(9), 1069-1076.

Guo, J., \& Li, B. (2018). The Application of Medical Artificial Intelligence Technology in Rural Areas of Developing Countries. Health equity, 2(1), 174-181. doi:10.1089/heq.2018.0037

Hamet, P., \& Tremblay, J. (2017). Artificial intelligence in medicine. Metabolism,69.

Harris, J. (2018). Altruism: Should it be included as an attribute of medical professionalism? Health Professions Education, 4(1), 3-8. doi:10.1016/j.hpe.2017.02.005

Heale R, Twycross A. (2015). Validity and reliability in quantitative studies. Evidence-Based Nursing ,18:66-67.

Holden, R. J., \& Karsh, B. T. (2009). The technology acceptance model: its past and its future in health care. Journal of biomedical informatics, 43(1), 159-172. doi:10.1016/j.jbi.2009.07.002

Holm, S. (2006). The WMA on Medical Ethics: Some Critical Comments. Journal of Medical Ethics, 32(3), 161-162. 
Holzinger, Andreas \& Malle, Bernd \& Kieseberg, Peter \& Roth, Peter M. \& Müller, Heimo \& Reihs, Robert \& Zatloukal, Kurt. (2017). Towards the Augmented Pathologist: Challenges of Explainable-AI in Digital Pathology.

Hoogland J, Jochemson H. Professional autonomy and the normative structure of medical practice. Theor Med Bioeth . 2000; 21:457-475.

Hosny, A., Parmar, C., Quackenbush, J., Schwartz, L. H., \& Aerts, H. (2018). Artificial intelligence in radiology. Nature reviews. Cancer, 18(8), 500-510. https://doi.org/10.1038/s41568-018-0016-5

Houghton, C., Murphy, K., Meehan, B., Thomas, J., Brooker, D. and Casey, D. (2017), From screening to synthesis: using NVIVO to enhance transparency in qualitative evidence synthesis. J Clin Nurs, 26: 873-881.

Howell LP. (2017). Making Artificial Intelligence Real in Pathology and Lab Medicine. Pathology chairs blog. UC Davis Health. https://blog.ucdmc.ucdavis.edu/pathologychair/index.php/2018/02/01/making-artificial-intelligence-real-in-pathology-and-lab-medicine/

Jay J. Ye (2015) Artificial Intelligence for Pathologists Is Not Near- It Is Here: Description of a Prototype That Can Transform How We Practice Pathology Tomorrow. Archives of Pathology \& Laboratory Medicine: July 2015, Vol. 139, No. 7, pp. 929-935

Jeffrey D. Goldsmith, Gene P. Siegal, Saul Suster, Thomas M. Wheeler, Richard W. Brown. (2008). Reporting Guidelines for Clinical Laboratory Reports in Surgical Pathology. Arch Pathol Lab Med. 2008;132:1608-1616. 
Jiang F, Jiang Y, Zhi H, et al. (2017). Artificial intelligence in healthcare: past, present and future. Stroke and Vascular Neurology;2: e000101.

Johnston, S. C. (2018). Anticipating and Training the Physician of the Future. Academic Medicine,93(8), 1105-1106.

Jones, R. (2002). Declining altruism in medicine. BMJ : British Medical Journal, 324(7338), 624.

Jung Rho, Mi \& Choi, Inyoung \& Lee, Jaebeom. (2014). Predictive factors of telemedicine service acceptance and behavioral intention of physicians. International Journal of Medical Informatics. 83. 10.1016/j.ijmedinf.2014.05.005.

Kayser, K. (2012). Introduction of virtual microscopy in routine surgical pathology - a hypothesis and personal view from Europe. Diagn Pathol 7, 48.

Kenny, B., Lincoln, M., \& Balandin, S. (2010). Experienced speech-language pathologists' responses to ethical dilemmas: an integrated approach to ethical reasoning. American journal of speech-language pathology, 19 2, 121-34 .

Keskinbora, K. H. (2019). Medical ethics considerations on artificial intelligence. Journal of Clinical Neuroscience : Official Journal of the Neurosurgical Society of Australasia, 64, 277-282. doi:10.1016/j.jocn.2019.03.001

Kolachalama, V. B., \& Garg, P. S. (2018). Machine learning and medical education. Npj Digital Medicine,1(1). doi:10.1038/s41746-018-0061-1

Larosa, E., \& Danks, D. (2018). Impacts on Trust of Healthcare AI. Proceedings of the 2018 AAAI/ACM Conference on AI, Ethics, and Society - AIES 18. doi:10.1145/3278721.3278771 
Laï, M., Brian, M. \& Mamzer, M. Perceptions of artificial intelligence in healthcare: findings from a qualitative survey study among actors in France. J Transl Med 18, 14 (2020).

Lazuras, L., \& Dokou, A. (2016). Mental health professional's acceptance of online counseling. Technology in Society,44, 10-14.

Lee Y-S, Song H-S, Kim H, Chae Y (2019) Altruistic decisions are influenced by the allocation of monetary incentives in a pain-sharing game. PLoS ONE 14(3): e0213104.

Li, J., Dow, W. H., \& Kariv, S. (2017). Social preferences of future physicians. Proceedings of the National Academy of Sciences of the United States of America, 114(48), E10291-E10300. doi:10.1073/pnas.1705451114

Lin, C., Lin, I., \& Roan, J. (2012). Barriers to physicians' adoption of healthcare information technology: An empirical study on multiple hospitals. Journal of Medical Systems, 36(3), 19651977.7

Lu, J. (2016). Will Medical Technology Deskill Doctors? International Education Studies,9(7), 130. doi:10.5539/ies.v9n7p130

Maher, C., Hadfield, M., Hutchings, M., \& de Eyto, A. (2018). Ensuring Rigor in Qualitative Data Analysis: A Design Research Approach to Coding Combining NVivo With Traditional Material Methods. International Journal of Qualitative Methods.

Manca D. P. (2015). Do electronic medical records improve quality of care? Yes. Canadian family physician Medecin de famille canadien, 61(10), 846-851. 
Moraligil, M., Bozdemir, M. N., İz, M., Layik, M. E., İz, F.,Betul Ay, \& Karaman, K. (2018). Determination of the knowledge and skills of physicians working in emergency services against judicial incidents. Eastern Journal of Medicine, 23(4), 247.

Nabuya Hashimoto. (2006). Professional Autonomy. Japan Medical Association journal: JMAJ, 49(3),125-127.

Noorbakhsh-Sabet N, Zand R, Zhang Y, Abedi V. (2019). Artificial Intelligence Transforms the Future of Health Care. Am J Med. 2019 Jul;132(7):795-801.

Nordin, I. (2000). Expert and non-expert knowledge in medical practice. Medicine, Health Care, and Philosophy, 3(3), 297-304.

Norman, I. D., Aikins, M. K., \& Binka, F. N. (2011). Ethics and electronic health information technology: challenges for evidence-based medicine and the physician-patient relationship. Ghana medical journal, 45(3), 115-124.

Ozair, F. F., Jamshed, N., Sharma, A., \& Aggarwal, P. (2015). Ethical issues in electronic health records: A general overview. Perspectives in clinical research, 6(2), 73-76. doi:10.4103/22293485.153997

Ozgur, C., Kleckner, M., \& Li, Y. (2015). Selection of Statistical Software for Solving Big Data Problems: A Guide for Businesses, Students, and Universities. SAGE Open.

Page, K. (2012). The four principles: Can they be measured, and do they predict ethical decision making? BMC Medical Ethics, 13(1), 10-10.

Panch, T., Mattie, H. \& Celi, L.A. (2019). The "inconvenient truth" about AI in healthcare. npj Digit. Med. 2, 77. 
Petronio, S., Dicorcia, M. J., \& Duggan, A. (2012). Navigating ethics of physician-patient confidentiality: a communication privacy management analysis. The Permanente journal, 16(4), $41-45$.

Plebani, M., Laposata, M., \& Lippi, G. (2019). A manifesto for the future of laboratory medicine professionals. Clinica Chimica Acta, 489, 49-52.

Phichitchaisopa, N., \& Naenna, T. (2013). Factors affecting the adoption of healthcare information technology. EXCLI journal, 12, 413-436.

Pouyan Esmaeilzadeha, Murali Sambasivanb,*, Naresh Kumarc, Hossein Nezakatid.(2015). Adoption of clinical decision support systems in a developing country: Antecedents and outcomes of physician's threat to perceived professional autonomy. International journal of medical informatics, 3181 .

Recht, M., \& Bryan, R. N. (2017). Artificial intelligence: Threat or boon to Radiologists? Journal of the American College of Radiology, 14(11), 1476-1480.

Reihs, Robert \& Zatloukal, Kurt. (2017). Towards the Augmented Pathologist: Challenges of Explainable-AI in Digital Pathology.

Salvatore, D., Numerato, D., \& Fattore, G. (2018). Physicians' professional autonomy and their organizational identification with their hospital. BMC Health Services Research,18(1).

Salla, E., Pikkarainen, M., Leväsluoto, J., Blackbright, H., \& Johansson, P. E. (2018). AI innovations and their impact on healthcare and medical expertise. Manchester: The International Society for Professional Innovation Management (ISPIM) 
Sambasivan, M., Esmaeilzadeh, P., Kumar, N., \& Nezakati, H. (2012). Intention to adopt clinical decision support systems in a developing country: effect of physician's perceived professional autonomy, involvement and belief: a cross-sectional study. BMC medical informatics and decision making, 12, 142 .

Scott A. Wolla, "Will Robots Take Our Jobs?" Page One Economics®, January 2018

Serag, A., Ion-Margineanu, A., Qureshi, H., McMillan, R., Saint Martin, M. J., Diamond, J., O'Reilly, P., \& Hamilton, P. (2019). Translational AI and Deep Learning in Diagnostic Pathology. Frontiers in medicine, 6, 185.

Skår, R. (2010), The meaning of autonomy in nursing practice. Journal of Clinical Nursing, 19: 2226-2234.

Baron D.N.(1992). Ethical problems in clinical pathology. J App Philosophy. 9;189 202.

Stoddard, J. J., Hargraves, J. L., Reed, M., \& Vratil, A. (2001). Managed care, professional autonomy, and income: effects on physician career satisfaction. Journal of general internal medicine, 16(10), 675-684.

Tammaro, D., Arrighi, J., Hilliard, R., \& Toll, E. (2018). Altruism: A story in three acts. The American Journal of Medicine, 131(12), 1526-1527.

Tarentines, K. (2014). Retention of knowledge and skills after advanced cardiovascular life support courses. American Journal of Emergency Medicine, 32(9), 1143-1147.

Tezuka K. (2014). Physicians and professional autonomy. Japan Medical Association journal: JMAJ, 57(3), 154-158. 
Tizhoosh HR, Pantanowitz L. (2018). Artificial intelligence and digital pathology: Challenges and opportunities. J Pathol Inform 2018; 9:38.

Timothy Craig Allen (2017) Twenty-First Century Pathologists' Advocacy. Archives of Pathology \& Laboratory Medicine: July 2017, Vol. 141, No. 7, pp. 940-943.

Tredinnick, L. (2017). Artificial intelligence and professional roles. Business Information Review, 34(1), 37-41.

Tyssen, R., Palmer, K. S., Solberg, I. B., Voltmer, E., \& Frank, E. (2013). Physicians' perceptions of quality of care, professional autonomy, and job satisfaction in Canada, Norway, and the United States. BMC health services research, 13, 516.

Vaughn, T. J. (2006). Psychology licensure and certification. American Psychological Association.

Walter, Z., \& Lopez, M. S. (2008). Physician acceptance of information technologies: Role of perceived threat to professional autonomy. Decision Support Systems,46(1), 206-215.

Walczak, Steven. (2018). The Role of Artificial Intelligence in Clinical Decision Support Systems and a Classification Framework. International Journal of Computers in Clinical Practice. 3. 31-47. 10.4018/IJCCP.2018070103.

Wong, S. T. (2018), Is pathology prepared for the adoption of artificial intelligence? Cancer Cytopathology, 126: 373-375.

Xue, Y., Liang, H., Mbarika, V., Hauser, R., Schwager, P., \& Kassa Getahun, M. (2015). Investigating the resistance to telemedicine in ethiopia. International Journal of Medical Informatics. 
Yang, C., Yen, Z., McGowan, J. E., Chen, H. C., Chiang, W., Mancini, M. E., . . Ma, M. H. (2012). A systematic review of retention of adult advanced life support knowledge and skills in healthcare providers. Resuscitation, 83(9), 1055-1060.

Young, P. (2017). Professional Autonomy. World Neurosurg, 104:983-984. 